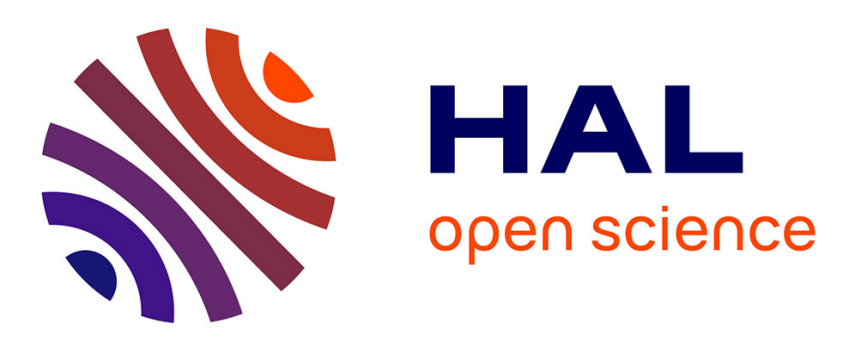

\title{
Interactions by exchange of volume between two spherical bubbles
}

Mahamadou Adama Maiga, Olivier Coutier Delgosha, Daniel Buisine

\section{To cite this version:}

Mahamadou Adama Maiga, Olivier Coutier Delgosha, Daniel Buisine. Interactions by exchange of volume between two spherical bubbles. Imperial College Press, 2014, 6 (6), pp.1450071. 10.1142/S1758825114500719 . hal-01276075

\section{HAL Id: hal-01276075 \\ https://hal.science/hal-01276075}

Submitted on 13 Nov 2017

HAL is a multi-disciplinary open access archive for the deposit and dissemination of scientific research documents, whether they are published or not. The documents may come from teaching and research institutions in France or abroad, or from public or private research centers.
L'archive ouverte pluridisciplinaire HAL, est destinée au dépôt et à la diffusion de documents scientifiques de niveau recherche, publiés ou non, émanant des établissements d'enseignement et de recherche français ou étrangers, des laboratoires publics ou privés. 


\title{
INTERACTIONS BY EXCHANGE OF VOLUME BETWEEN TWO SPHERICAL BUBBLES
}

\author{
M. ADAMA MAIGA*, ${ }^{*}$ O. COUTIER-DELGOSHA* and D. BUISINE ${ }^{\dagger}$ \\ *LML Laboratory (Laboratoire de Mécanique de Lille)/ \\ Arts et Metiers ParisTech, 8 Boulevard Louis \\ XIV, 59046 Lille, France \\ ${ }^{\dagger}$ LML Laboratory (Laboratoire de Mécanique de Lille)/ \\ Université des Sciences et Technologies de \\ Lille, Mechanic Department \\ 59650 Villeneuve d'Ascq, France \\ ${ }^{\ddagger}$ Mahamadou.adamamaiga@ensam.eu
}

Received 19 February 2014

Revised 11 July 2014

Accepted 29 July 2014

Published 17 November 2014

\begin{abstract}
In this paper, by using the system potential of two bubbles and with a special interest in the interaction by exchange of volume and without exchange of mass, a system of equations governing the evolution of two bubbles is proposed. This two-bubble model shows terms that do not appear in the models of interaction between bubbles. The twobubble model is compared with the modified Rayleigh-Plesset equation and a validation with the experimental study of $\mathrm{Ohl}$ [2002] is presented. The numerical results show that, on one hand, the development of small nearby bubbles can slow down the evolution of the biggest local one, while their disappearance can favor its growing. Furthermore, in the case of two bubbles in particular, the small bubble exchanges volume with the big one during their evolutions. On the other hand, contrary to the modified Rayleigh-Plesset model, the two-bubble model predicts appearance and disappearance of small bubbles in the neighborhood of the big bubble as it is observed in the experimental study of Ohl [2002].

The present findings show in particular that the interaction by exchange of volume can be very important in the cavitation born phase and it is necessary to take into account the interaction between bubbles as well as the disappearance of small ones on the evolution of the biggest local bubble. Also, this two-bubble model predicts an exchange of volume between both bubbles equal to zero when they are perfectly identical.
\end{abstract}

Keywords: Cavitation; modeling; multibubble; interactions; exchange of volume.

\section{Introduction}

The study of phase change phenomena and more in particular cavitation, in which vapor bubbles are formed in a liquid under pressure drop effect, still remains a challenge in mechanics.

Experimental studies [Lauterborn and Bolle, 1975; Franc and Michel, 1985; Arndt et al., 1989; Madadnia and Owen, 1995; Astolfi et al., 2000; Fortes-Patella 
et al., 2000; Ohl, 2002; Stutz and Legoupil, 2003; Bremond et al., 2006; CoutierDelgosha et al., 2007; Dular et al., 2012] allowed us to move forward in the understanding of the inception, the development and the structure of cavitation as well as the erosion caused by this phenomenon. More studies [Ohl, 2002; Buogo and Cannelli, 2002; Abe et al., 2007; Yang et al., 2009; Aghdam et al., 2012] were carried out to comprehend the inception and the evolution of one single cavitation bubble. However, even in these cases, the single bubble is mostly formed after the coalescence of a multitude of small bubbles that appear in the early stage of the bubble inception. These premature disappearances of small bubbles can influence the evolution and growth of the final bubble. In the experimental study of the cavitation inception, Ohl [2002] concluded that: the observed scenario suggests that the bubble-bubble interactions have to be taken into account for accurate modeling. Then, a simple ansatz using a single-bubble model is not valid.

The first theoretical studies on the evolution of single-bubble cavitation were led by Rayleigh [1917]. The Rayleigh equation was developed by Plesset [1948] and is known by Rayleigh-Plesset equation. Other equations of single-bubble radial evolution exists [Gilmore, 1952; Keller and Miksis, 1980].

In the case of two or several bubbles, many studies based on bubbles cloud and interactions between bubbles were carried out [Chahine, 1984; d'Agostino and Brennen, 1989; Oguz and Prosperetti, 1990; Kubota et al., 1992; Takahira et al., 1994; Mettin et al., 1997; Harkin et al., 2001; Bremond et al., 2005; Ida, 2007].

Some multibubble models are based on the modified single-bubble equations. In fact, these equations are modified to take into account the nearby bubbles influence. The added terms are functions of the interbubble distances $D_{i j}$, which represents the distance between the centers of the bubbles $i$ and $j$. Also, a great majority of two or more bubbles make an approximation of the system potential in the neighborhood of each bubble.

In this study, the idea is to take into account the premature disappearance of small bubbles at the initial stage of cavitation inception, which can contribute by exchange of volume without exchange of mass to the growing of a small number of big bubbles.

In a physical phenomenon study, the understanding of a particular case mostly allows to move forward in the understanding of the general case. So, in the present work, we are interested in the interaction by exchange of volume without exchange of mass. We consider two bubbles separated by the distance $D_{12}$, the bubbles translate velocities induced by their mutual interactions are neglected, with specific hypothesis, the system potential will be the sum of the potential bubble. This potential allows to obtain the system velocity field. With the unidimensional equation of Navier-Stokes and an interest in the interaction by exchange of volume, the equation governing the evolution of both bubbles is obtained. The equation shows terms which do not appear in most of interaction models between bubbles. For the validation, the model is compared with the modified Rayleigh-Plesset equation and 
the experimental study of Ohl [2002]. The latter is one of rare experimental studies in which the pressure and the radius of the cavitation bubbles are both measured as a function of time at cavitation inception.

Our work is divided into two parts. In Sec. 1, the modified Rayleigh-Plesset equation model is introduced and the two-bubble model is developed. The second section is dedicated to the comparison of results from each model as well as the validation with experimental study.

\section{Bubbles Evolution Models}

Studies [Oguz and Prosperitti, 1990; Harkin et al., 2001; Doinikov, 2001] show that in the case where the interbubble distance $D_{i j}$ is much bigger than the initial bubbles radii, the translate velocities of bubbles variation can be very low. Bremond et al. [2006] showed that the modified Rayleigh-Plesset equation for two bubbles describes well the instantaneous bubble radii evolution until it reaches $75 \%$ of half the distance $D_{i j}$. In this present work, $D_{i j}$ remains constant and $\left(R_{i}+R_{j}\right)$ is inferior than $0.75 \times D_{i j}\left(\left(R_{i}+R_{j}\right) \leq 0.75 D_{i j}\right)$. The same hypothesis has been also used by Ida [2009], $D_{i j}$ was considered constant (the order of $D_{i j} \geq D_{0}\left(R_{0 i}+R_{0 j}\right)$ with $\left.D_{0}=10\right)$.

\subsection{Multibubble cavitation model}

For the multibubble model, the modified Rayleigh-Plesset equation [Bremond et al., 2006; Ida, 2009] is used. With $N_{b}$ the number of bubbles, this equation, rewritten with the variable flow rate, is:

$$
\dot{q}_{i}=\frac{1}{8 \pi} \frac{q_{i}^{2}}{R_{i}^{3}}-\frac{4 \mu_{L}}{\rho_{L}} \frac{q_{i}}{R_{i}^{2}}+\frac{4 \pi R_{i}}{\rho_{L}}\left(p_{g_{i}}+p_{v}-p_{\mathrm{ex}}(t)\right)-\frac{8 \pi \sigma}{\rho_{L}}-\sum_{j=1, j \neq i}^{N_{b}} \frac{R_{i}}{D_{i j}} \dot{q}_{j}
$$

where $D_{i j}$ is the distance between the centers of bubbles $i$ and $j, R_{i}$ is the radius, $\dot{R}_{i}$ is the normal velocity to the interface, $q_{i}=4 \pi R_{i}^{2} \dot{R}_{i}$ the flow rate, $\dot{q}_{i}=\frac{\partial q_{i}}{\partial t}=$ $4 \pi\left(2 R_{i} \dot{R}_{i}^{2}+R_{i}^{2} \ddot{R}_{i}\right)$, and $\dot{q}_{j}=\frac{\partial q_{j}}{\partial t}=4 \pi\left(2 R_{j} \dot{R}_{j}^{2}+R_{j}^{2} \ddot{R}_{j}\right), \sigma$ is the surface tension, $\mu_{L}$ the dynamic viscosity, $\rho_{L}$ is density of liquid, $p_{i}$ is the pressure in the liquid at the bubble interface. The pressure inside bubbles equals to the sum of the vapor pressure $p_{v}$ and the gas pressure $p_{g_{i}}$, modeled by the barotropic Laplace law:

$$
p_{g_{i}}=\left(p_{0}+\frac{2 \sigma}{R_{0 i}}-p_{v}\right)\left(\frac{R_{0 i}}{R_{i}}\right)^{3 \gamma}
$$

where $R_{0 i}$ is the initial radius, $\gamma$ is the polytropitic exponent, $p_{0}$ is the atmospheric pressure and $p_{\text {ex }}(t)$ is the ambient environment pressure.

\subsection{Two-bubble model}

In the two-bubble model, two isolated bubbles are considered. From now on, the index 1 will be allocated to the big bubble and the index 2 to the small one: Fig. 1. 


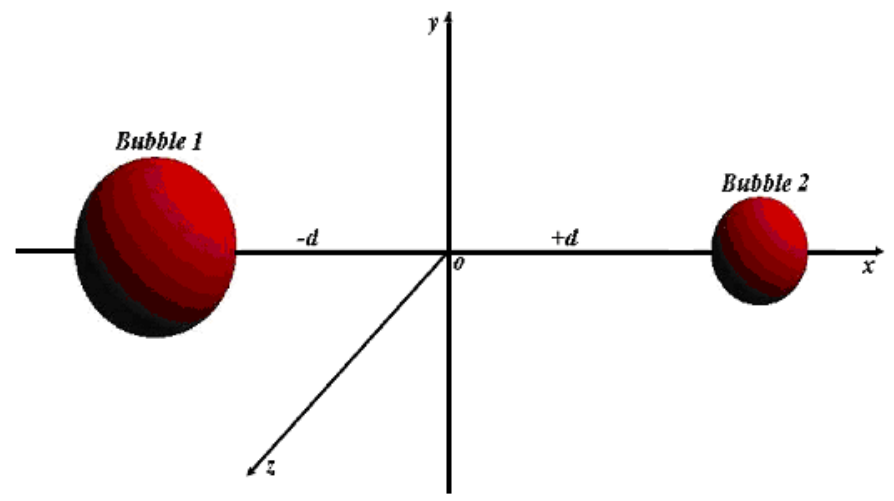

Fig. 1. Schematic position of the two bubbles.

Both bubbles are assumed to remain spherical, the vorticity is assumed to be zero, the liquid flow is supposed to be potential, and bubbles can be considered as sources. The condition of sliding is thus not respected exactly. If the bubbles translation velocity is neglected, the potential of the system will simply be the sum of the separate potentials due to bubbles

$$
F(x, y, z)=-\frac{1}{4 \pi}\left(\frac{q_{1}}{\left\|X_{F}-X_{1}\right\|}+\frac{q_{2}}{\left\|X_{F}-X_{2}\right\|}\right)
$$

where $X_{F}=(x, y, z), X_{1}=(-d, 0,0), X_{2}=(d, 0,0)$ and $D_{12}=2 d . q_{1}=4 \pi R_{1}^{2} \dot{R}_{1}$ and $q_{2}=4 \pi R_{2}^{2} \dot{R}_{2}$ are the expansion flow rate of bubbles 1 and $2 . R_{1}$ and $R_{2}$ are their radii, respectively.

With Eq. (3), $\mathbf{v}=\nabla F(x, y, z)$, the velocity of the fluid in $x$ direction between the centers is

$$
v(x)=\frac{1}{4 \pi}\left(\frac{q_{1}}{(d+x)^{2}}-\frac{q_{2}}{(d-x)^{2}}\right) .
$$

With $-d+R_{1}<x<d-R_{2}$. Because potential flow is considered in this approach, the viscosity term in the Navier-Stokes equations is equal to zero. Thus, the NavierStokes equation in the $x$ direction can be written as

$$
\frac{\partial v}{\partial t}+v \frac{\partial v}{\partial x}=-\frac{1}{\rho_{L}} \frac{\partial p}{\partial x}
$$

where $p$ is the local static pressure and $\rho_{L}$ is the density of the liquid. By integrating this equation and taking into account the expression of the velocity at the interface of the bubble $i: v_{i}=\dot{R}_{i}=\frac{q_{i}}{4 \pi R_{i}^{2}}$, with $\dot{R}_{i}=\frac{\partial R_{i}}{\partial t}$ :

$$
\frac{1}{4 \pi}\left[\frac{\dot{q}_{1}}{(d+x)}+\frac{\dot{q}_{2}}{(d-x)}\right]_{x_{1}}^{x_{2}}=\left[\frac{p}{\rho_{L}}+\frac{1}{32 \pi^{2}}\left(\frac{q_{1}}{(d+x)^{2}}-\frac{q_{2}}{(d-x)^{2}}\right)^{2}\right]_{x_{1}}^{x_{2}}
$$


with $x_{1}=-d+R_{1}$ and $x_{2}=d-R_{2}$.

$$
\begin{aligned}
& \frac{1}{4 \pi}\left(\frac{\dot{q}_{1}}{2 d-R_{2}}+\frac{\dot{q}_{2}}{R_{2}}-\frac{\dot{q}_{1}}{R_{1}}-\frac{\dot{q}_{2}}{2 d-R_{1}}\right) \\
& \quad=\frac{1}{32 \pi^{2}}\left[\left(\frac{q_{1}}{\left(2 d-R_{2}\right)^{2}}-\frac{q_{2}}{R_{2}^{2}}\right)^{2}-\left(\frac{q_{1}}{R_{1}^{2}}-\frac{q_{2}}{\left(2 d-R_{1}\right)^{2}}\right)^{2}\right]+\frac{p_{2}-p_{1}}{\rho_{L}}
\end{aligned}
$$

It follows:

$$
\begin{aligned}
\frac{\dot{q}_{1}}{4 \pi R_{1}}-\frac{q_{1}^{2}}{32 \pi^{2} R_{1}^{4}}-\frac{p_{1}}{\rho_{L}}= & \frac{\dot{q}_{1}}{4 \pi\left(D_{12}-R_{2}\right)}-\frac{\dot{q}_{2}}{4 \pi\left(D_{12}-R_{1}\right)}-\frac{q_{1}^{2}}{32 \pi^{2}\left(D_{12}-R_{2}\right)^{4}} \\
& +\frac{q_{2}^{2}}{32 \pi^{2}\left(D_{12}-R_{1}\right)^{4}}+\frac{q_{1} q_{2}}{16 \pi^{2} R_{1}^{2} R_{2}^{2}}\left(\frac{R_{1}^{2}}{\left(D_{12}-R_{2}\right)^{2}}\right. \\
& \left.-\frac{R_{2}^{2}}{\left(D_{12}-R_{1}\right)^{2}}\right)+\frac{\dot{q}_{2}}{4 \pi R_{2}}-\frac{q_{2}^{2}}{32 \pi^{2} R_{2}^{4}}-\frac{p_{2}}{\rho_{L}}
\end{aligned}
$$

with $\dot{q}_{1}=\frac{\partial q_{1}}{\partial t}=4 \pi\left(2 R_{1} \dot{R}_{1}^{2}+R_{1}^{2} \ddot{R}_{1}\right), \dot{q}_{2}=\frac{\partial q_{2}}{\partial t}=4 \pi\left(2 R_{2} \dot{R}_{2}^{2}+R_{2}^{2} \ddot{R}_{2}\right), p_{1}$ and $p_{2}$ are pressures in the liquid at the interface of bubbles 1 and 2. Equation (8) governs their evolution. One can note that the ambient environment pressure does not appear in Eq. (8) and by considering two quasi-identical bubbles, the functions of $D_{12}$ terms, which govern particularly the interactions between bubbles, neutralize each other. In this case, the interaction by exchange of volume is nil, as all other interactions are supposed negligible, the evolution of each bubble is governed only by the ambient environment pressure. So, the ambient environment pressure can be estimated by

$$
-\frac{p_{\mathrm{ex}}(t)}{\rho_{L}} \approx \frac{\dot{q}_{2}}{4 \pi R_{2}}-\frac{q_{2}^{2}}{32 \pi^{2} R_{2}^{4}}-\frac{p_{2}}{\rho_{L}}
$$

where $p_{\text {ex }}(t)$ the ambient environment pressure. Equation (9) is the classic RayleighPlesset equation rewritten with the variable flow rate. In this case however, it only allows to estimate the ambient environment pressure and not the evolution of the bubble.

By considering Eqs. (8) and (9), we obtain the equation governing the evolution of bubble 1

$$
\begin{aligned}
\frac{\dot{q}_{1}}{4 \pi R_{1}} & \left(1-\frac{R_{1}}{D_{12}-R_{2}}\right)-\frac{q_{1}^{2}}{32 \pi^{2} R_{1}^{4}}\left(1-\frac{R_{1}^{4}}{\left(D_{12}-R_{2}\right)^{4}}\right) \\
& -\frac{q_{1} q_{2}}{16 \pi^{2} R_{1}^{2} R_{2}^{2}}\left(\frac{R_{1}^{2}}{\left(D_{12}-R_{2}\right)^{2}}-\frac{R_{2}^{2}}{\left(D_{12}-R_{1}\right)^{2}}\right) \\
= & \frac{p_{1}}{\rho_{L}}-p_{\text {ext }}(t)-\frac{\dot{q}_{2}}{4 \pi\left(D_{12}-R_{1}\right)}+\frac{q_{2}^{2}}{32 \pi^{2}\left(D_{12}-R_{1}\right)^{4}} .
\end{aligned}
$$


The equation which governs the evolution of bubble 2 is

$$
\begin{aligned}
\frac{\dot{q}_{2}}{4 \pi R_{2}} & \left(1-\frac{R_{2}}{D_{12}-R_{1}}\right)-\frac{q_{2}^{2}}{32 \pi^{2} R_{2}^{4}}\left(1-\frac{R_{2}^{4}}{\left(D_{12}-R_{1}\right)^{4}}\right) \\
& -\frac{q_{1} q_{2}}{16 \pi^{2} R_{1}^{2} R_{2}^{2}}\left(\frac{R_{2}^{2}}{\left(D_{12}-R_{1}\right)^{2}}-\frac{R_{1}^{2}}{\left(D_{12}-R_{2}\right)^{2}}\right) \\
= & \frac{p_{2}}{\rho_{L}}-p_{\text {ext }}(t)-\frac{\dot{q}_{1}}{4 \pi\left(D_{12}-R_{2}\right)}+\frac{q_{1}^{2}}{32 \pi^{2}\left(D_{12}-R_{2}\right)^{4}} .
\end{aligned}
$$

The equation of equilibrium at the interface of a bubble can be expressed as

$$
\begin{aligned}
& p_{1}=p_{v}+p_{g_{1}}-4 \mu_{L} \frac{\dot{R}_{1}}{R_{1}}-2 \frac{\sigma}{R_{1}} \\
& p_{2}=p_{v}+p_{g_{2}}-4 \mu_{L} \frac{\dot{R}_{2}}{R_{2}}-2 \frac{\sigma}{R_{2}} .
\end{aligned}
$$

By considering $R_{i}$ negligible compared to $D_{12}$, the terms $\frac{\dot{q}_{i}}{4 \pi D_{12}}$ and $\frac{q_{1} q_{2}}{16 \pi^{2} D_{12}}\left(\frac{1}{R_{2}^{2}}-\right.$ $\left.\frac{1}{R_{1}^{2}}\right)$ will not appear in the equation governing the evolution of the bubble $i$ in the majority of existing multibubble models.

We write

$$
q_{1}-q_{2}=q_{e}
$$

where $q_{e}$ is the exchange flow rate between bubbles 1 and 2 .

\section{Results and Discussions}

As mentioned earlier, the model validation is performed by comparing the results obtained from the present work to those obtained in the experimental study of Ohl [2002]. The measured evolution of the pressure equation $p_{\text {ex }}(t)$ is

$$
\begin{aligned}
p_{ \pm}= & \frac{1}{2}\left[p_{10} \exp \left(-\alpha_{1}\left(t \pm \frac{x}{c}\right)\right)+p_{20} \exp \left(-\alpha_{2}\left(t \pm \frac{x}{c}\right)\right)\right. \\
& \left.* \cos \left[\left(\sum_{i=0}^{3} \nu_{i}\left(\left(t \pm \frac{x}{c}\right)\right)^{i}\right) 2 \pi\left(t \pm \frac{x}{c}\right)+\phi\right]\right] \\
& *\left[1 .+\tanh \left(n_{1}\left(t \pm \frac{x}{c}\right)\right)\right] *\left[1 .+\tanh \left(n_{2}\left(\Delta-\left(t \pm \frac{x}{c}\right)\right)\right)\right]
\end{aligned}
$$

where $c$ is the speed of sound, other constants are given in Table 1.

Table 1. Values of constants in Eq. (13).

\begin{tabular}{lccc}
\hline$P_{01}=2.275 \cdot 10^{7} \mathrm{~Pa}$ & $P_{02}=2.77 \cdot 10^{6} \mathrm{~Pa}$ & $\alpha_{1}=1.767 \cdot 10^{6} \mathrm{~s}^{-1}$ & $\alpha_{2}=-9.987 \cdot 10^{4} \mathrm{~s}^{-1}$ \\
\hline$n_{1}=4.674 \cdot 10^{7} \mathrm{~s}^{-1}$ & $n_{2}=5 \cdot 10^{6} \mathrm{~s}^{-1}$ & $v_{0}=-3.21 \cdot 10^{3} \mathrm{~s}^{-1}$ & $v_{1}=2.16 \cdot 10^{10} \mathrm{~s}^{-2}$ \\
$v_{2}=-1.03 \cdot 10^{9} \mathrm{~s}^{-3}$ & $v_{3}=-1.08 \cdot 10^{7} \mathrm{~s}^{-4}$ & $\varphi=7.44$ & $\Delta=7 \cdot 10^{-6} \mathrm{~s}$ \\
\hline
\end{tabular}




\subsection{Large number of bubbles case}

The objective of this part is to quantify, with the classic multibubble model (the modified Rayleigh-Plesset equation), the influence of the nearby bubbles on the evolution of the local biggest one. We consider different configurations in both cases: orderly and disorderly bubbles distribution.

For the orderly distribution of bubbles, 3 configurations are tested: see Fig. 2. In configuration I, 5 bubbles are distributed on a square of side $C_{0}=\sqrt{2} D_{12}$. A bubble of initial radius $R_{01}=10 \mu \mathrm{m}$ is situated in the center and four of the same radius $(2 \mu \mathrm{m})$ are placed on each summit. This configuration can be considered as a case of two bubbles with radius $R_{1}$ and $R_{2}$.

In configuration II, 9 bubbles are distributed in a cube of edge $C_{0}=\frac{2}{\sqrt{3}} D_{12}$. A bubble of initial radius $R_{01}=10 \mu \mathrm{m}$ is located in the center of the cube and 8 of the same radius $(2 \mu \mathrm{m})$ are placed on each summit. This configuration II can also be considered as a case of two bubbles with radius $R_{1}$ and $R_{2}$.

In configuration III, we consider 15 bubbles distributed in a cube of edge $C_{0}=$ $2 D_{12}$. A bubble of initial radius $R_{01}=10 \mu \mathrm{m}$ is in the center of the cube, 6 bubbles of the same radius $(5 \mu \mathrm{m})$ are placed in the center of each face and 8 bubbles of the same radius $(2 \mu \mathrm{m})$ are on each summit. This configuration III can be considered as a case of three bubbles with radius $R_{1}, R_{2}$ and $R_{3}$.

For these three configurations, $D_{12}=D_{0}\left(R_{01}+R_{02}\right)$ with $D_{0}=10$. For the system of equations, see Appendix.

In the case of disorderly distribution of bubbles, the initial radius of the biggest bubble is set to $R_{01}=10 \mu \mathrm{m}$ and of the smallest one to $R_{0 N_{b}}=2 \mu \mathrm{m}$ where $N_{b} \geq 2$ is the number of bubbles. Radii of the other bubbles are determined by a geometrical continuation of reason $\left(R_{0 N_{b}} / R_{01}\right)^{1 /\left(N_{b}-1\right)}: R_{0 i}=\left(R_{0 N_{b}} / R_{01}\right)^{1 /\left(N_{b}-1\right)} R_{0 i-1}, i \geq 2$ and the distance between centers $i$ and $j$ is $D_{i j}=D_{0}\left(R_{0 i}+R_{0 j}\right)$ with $D_{0}=10$.

Figure 3 represents bubbles evolution in the three configurations of orderly bubbles distribution as well as the case of a single-bubble evolution.

The results show there is only small difference in big bubbles radii between configuration I and the case of only one single bubble, without any small bubble in the neighborhood. The big bubble in this configuration behaves almost like if

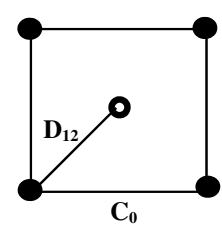

Confi. I

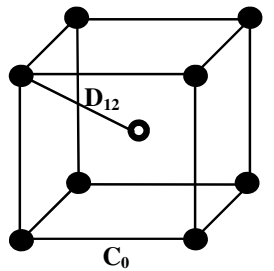

Confi. II

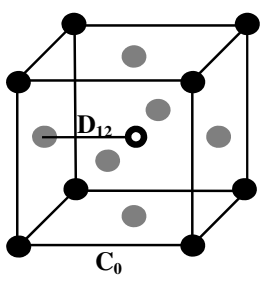

Confi. III

Fig. 2. Three configurations of orderly bubbles distribution. 

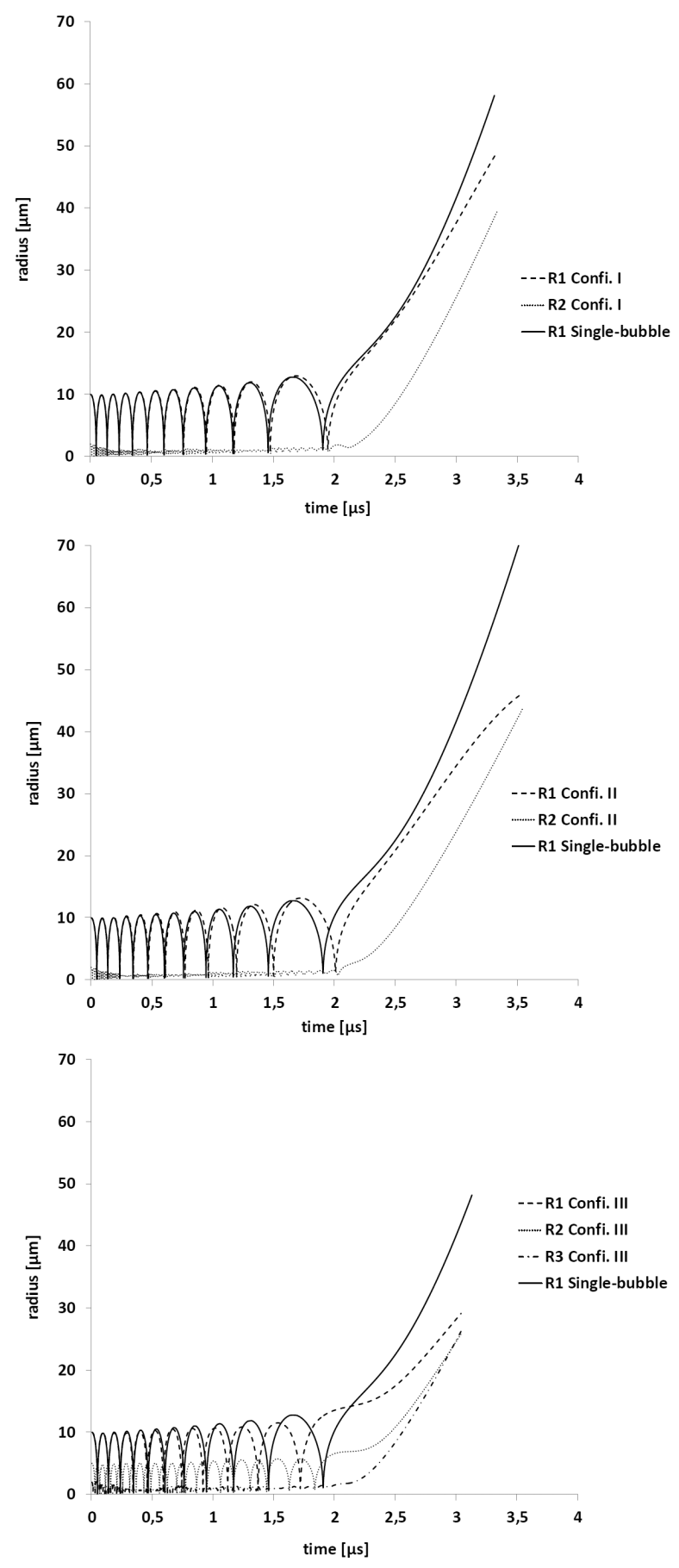

Fig. 3. Bubble evolution in configurations I, II and III and single-bubble evolution case. 


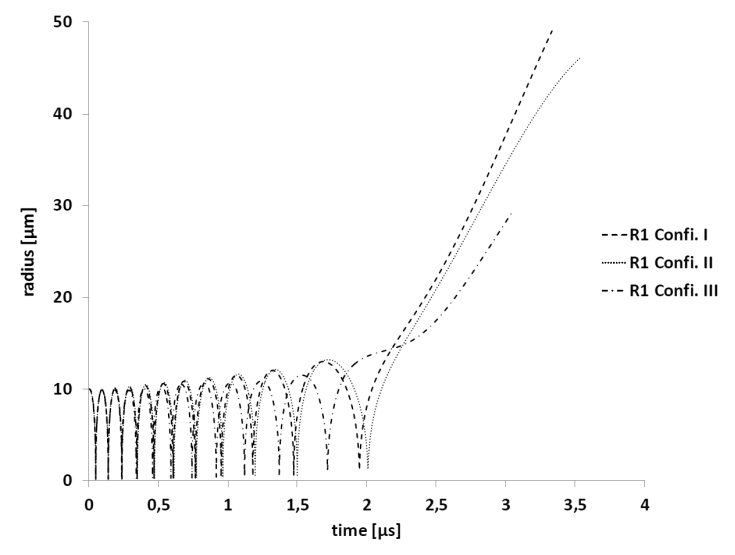

Fig. 4. Evolution of the radius $R_{1}$ in the configurations I, II and III.

it is alone and the interactions with the 4 small bubbles in the neighborhood are low. However, in configurations II (with 8 bubbles in the neighborhood) and III (14 bubbles in the neighborhood), the radii of the big bubbles are much smaller than the one of the single-bubble case.

Figure 4 shows that the radius $R_{1}$ in configuration $\mathrm{I}$ is bigger than the one in configuration II which is bigger than the one in configuration III.

The results of Figs. 3 and 4 indicate particularly that the more there are small growing bubbles in the neighborhood of the local big one $\left(R_{1}\right)$ the less this one develops. This finding can also be observed from Fig. 5 which represents the evolution of radius $R_{1}$ in configurations of disorderly distribution of bubbles: In this case $N_{b}$ is the number of bubbles and is equal to 4, 11 and 14 . However, the same figure shows that for $N_{b}=16$ the radius $R_{1}$ is bigger than in all other cases. This could be explained by disappearance of small bubbles when $N_{b}=16$. In fact, Fig. 6

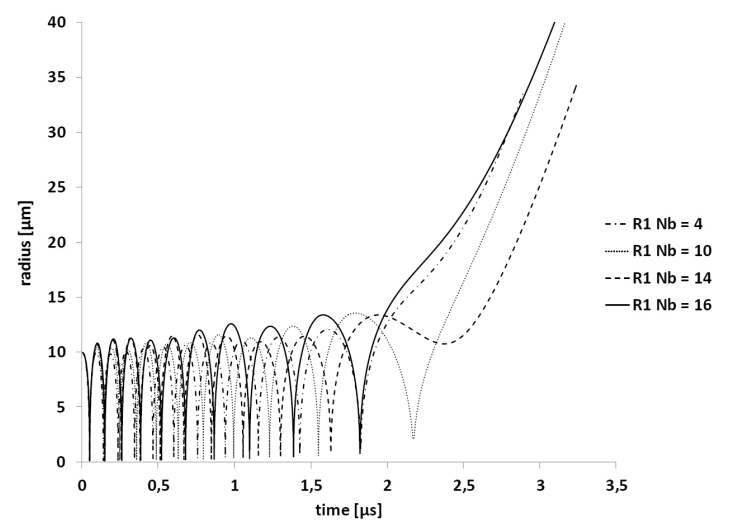

Fig. 5. Evolution of $R_{1}$ from the modified Rayleigh-Plesset model for $N_{b}$ equal to $4,11,14$ and 16 . 


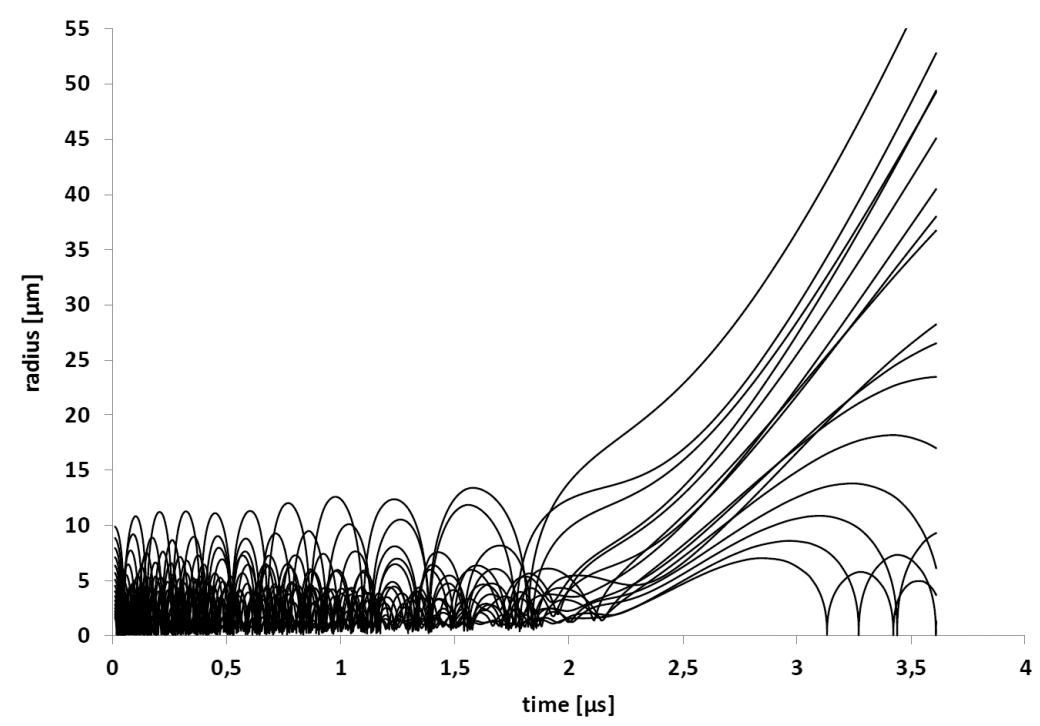

Fig. 6. Evolution of the radii from the modified Rayleigh-Plesset model for $N_{b}=16$.

represents the evolution of all 16 bubbles. This results show clearly that certain small bubbles disappear to let the local biggest one develop. Thus, it is important to take into account the disappearance of these small bubbles on the evolution of the big one.

From now on, we consider $N_{b}=2$ in the modified Rayleigh-Plesset model, this allows a better comparison with the two-bubble model. Also, in order to simplify the curve legends, a variable $Y$ obtained by the modified Rayleigh-Plesset model will be written as $Y$ MRP $D_{0}=X$ and the two-bubble one as $Y$ TBM $D_{0}=X$. Example, $R 1$ MRP $D_{0}=10$ represents the radius $R_{1}$ of the modified RayleighPlesset model for $D_{0}=10$ and $R 2$ TBM $D_{0}=10$ the radius $R_{2}$ of the two-bubble model for $D_{0}=10$.

\subsection{Comparison and validation of models}

For a better investigation, we consider two initial bubbles of almost the same size $R_{01}=10 \mu \mathrm{m}$ and $R_{02}=9.9 \mu \mathrm{m}$, this allows to have an exchange flow rate between bubbles almost equal to zero at the early moments.

Figure 7 represents, for different $D_{12}=D_{0}\left(R_{01}+R_{02}\right): D_{0}$ equal to 10,21 and 22 , the time evolution of the radii $R_{1}$ and $R_{2}$ of the modified Rayleigh-Plesset model. The results show that, for $D_{0}$ equal to 22 , both bubbles develop at almost the same size. For $D_{0}$ equal to 10 and 21, one can see a small difference between both radii, this difference is explained by the fact that at these distances the interactions between bubbles could be strong. This observation can clearly be seen in Fig. 8, which represents the evolution of the radii $R_{1}$ and $R_{2}$ of the two-bubble model. 

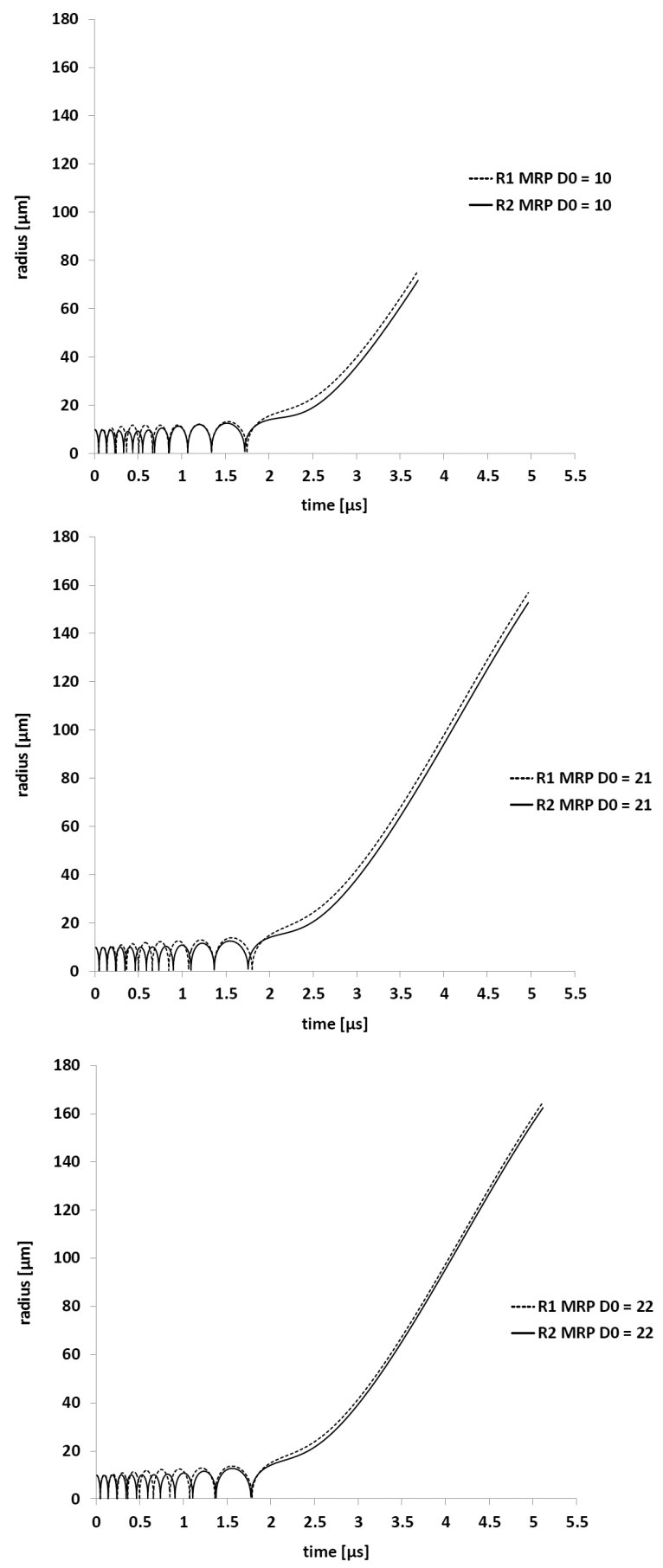

Fig. 7. Evolution of $R_{1}$ and $R_{2}$ from the modified Rayleigh-Plesset model for $D_{0}$ equal to 10,21 and 22 . 

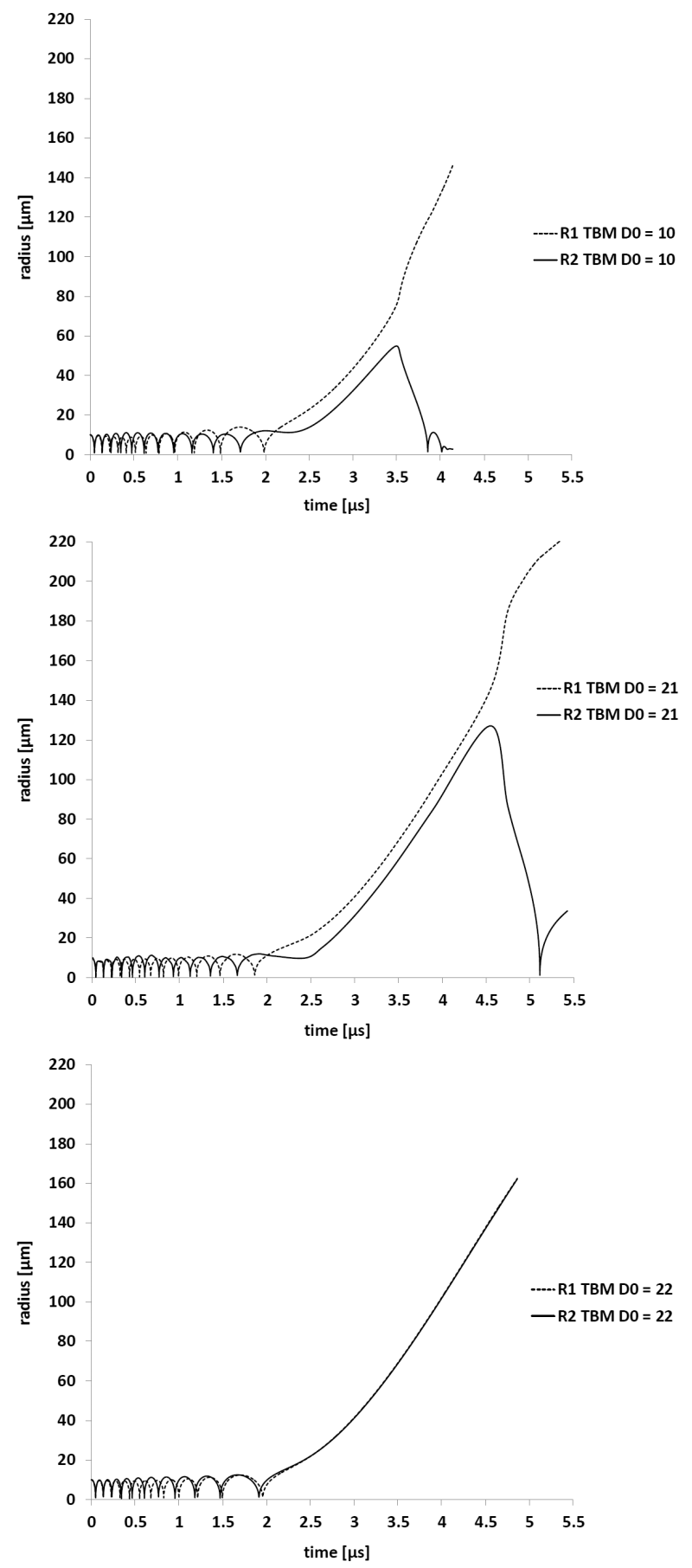

Fig. 8. Evolution of $R_{1}$ and $R_{2}$ from the two-bubble model for $D_{0}$ equal to 10,21 and 22 . 


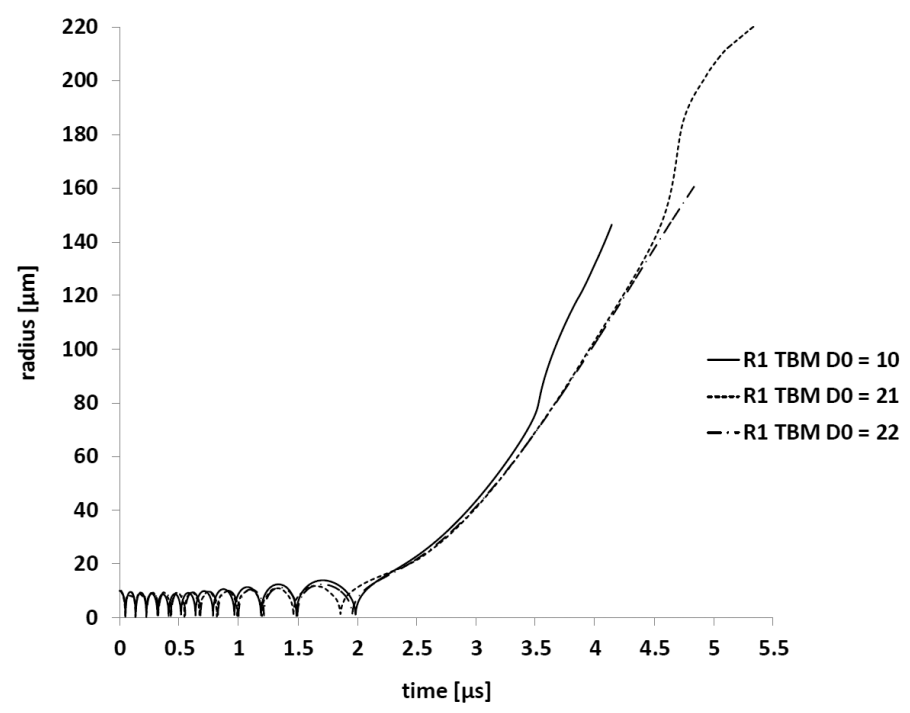

Fig. 9. Comparison of $R_{1}$ from the two-bubble model for $D_{0}$ equal to 10,21 and 22 .

One can see that for $D_{0}$ equal to 10 and 21, the small bubble develops at first, then collapses after a certain time. However, when $D_{0}$ is equal to 22 , as with the modified Rayleigh-Plesset model, both bubbles develop at almost the same size.

The influence of this collapse, or in a general way the interaction between bubbles, can be observed on one hand in Fig. 9 where one can see that the smaller $D_{0}$ is, the bigger $R_{1}$ gets. On the other hand, Fig. 10 shows that for different $D_{0}$, the radius $R_{1}$ of the two-bubble model is bigger than the one of the modified RayleighPlesset model. Figure 11 shows that the radius $R_{2}$ in the two-bubble model is less developed than in the modified Rayleigh-Plesset. However for $D_{0}$ equal to 22, both models produce almost the same radii $R_{1}$ and $R_{2}$. In this case, the interaction between bubbles is very low.

Interaction between both bubbles can be quantified as the exchange of volume or flow rate $q_{e}$ between them. Figure 12, which represents the time evolution of flow rate $q_{e}$ of the models for $D_{0}$ equal to 10,21 and 22 , is an illustration of models comparison. The results show that, for $D_{0}$ equal to 22 , the flow rate $q_{e}$ is very low for both models. Consequently, interactions between bubbles by exchange of volume are very low, and as indicated in these cases, both bubbles have almost the same size. Also, the results show that, for $D_{0}$ equal to 10 and 21, the interaction by exchange of volume in the two-bubble model is more important than the one in modified Rayleigh-Plesset model. Also, for the latter and for $D_{0}$ equal to 10 and 21, one can see that the interactions between bubbles are quite strong even if these interactions are weaker than for the two-bubble model. This justifies the differences between bubbles radii in Fig. 7 where two bubbles with initial radius of $9.9 \mu \mathrm{m}$ and $10 \mu \mathrm{m}$ were compared using modified Rayleigh-Plesset model. 

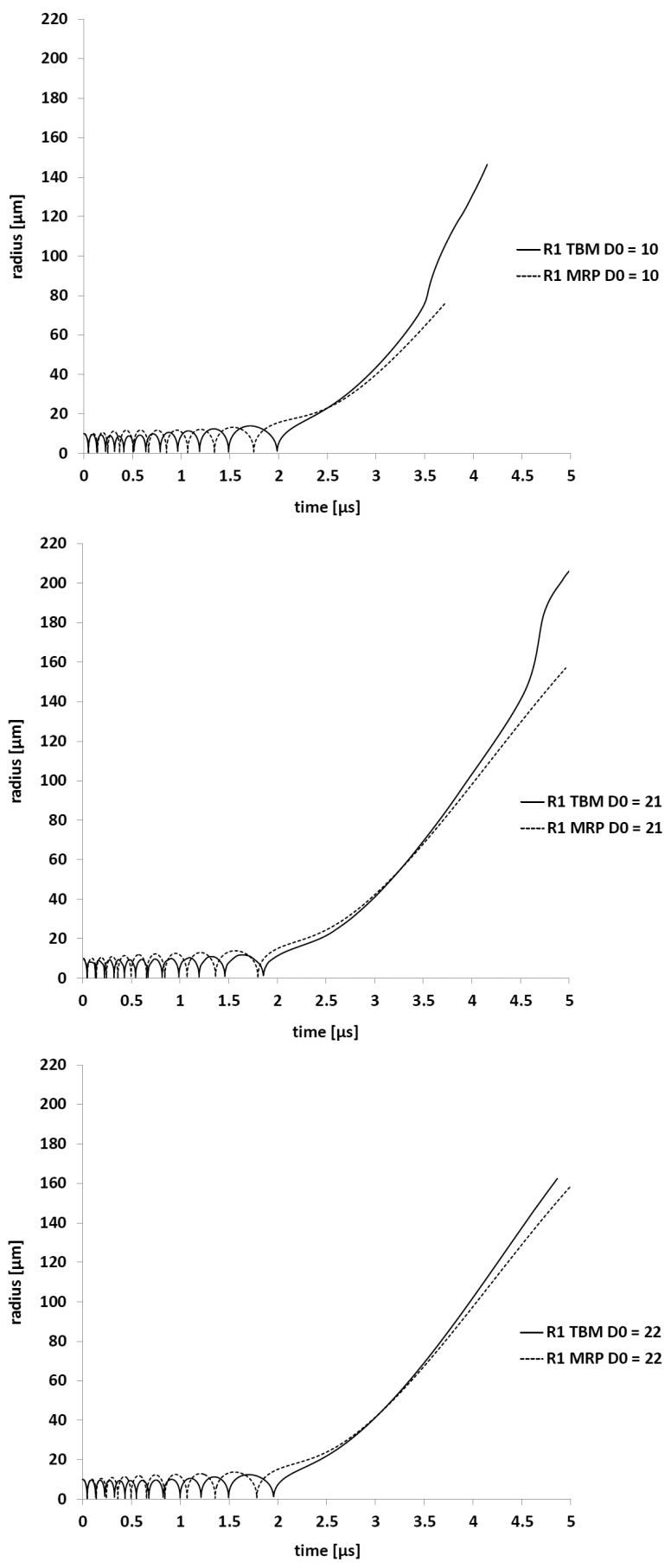

Fig. 10. Comparison of $R_{1}$ from the modified Rayleigh-Plesset model and the two-bubble model for $D_{0}$ equal to 10,21 and 22 . 

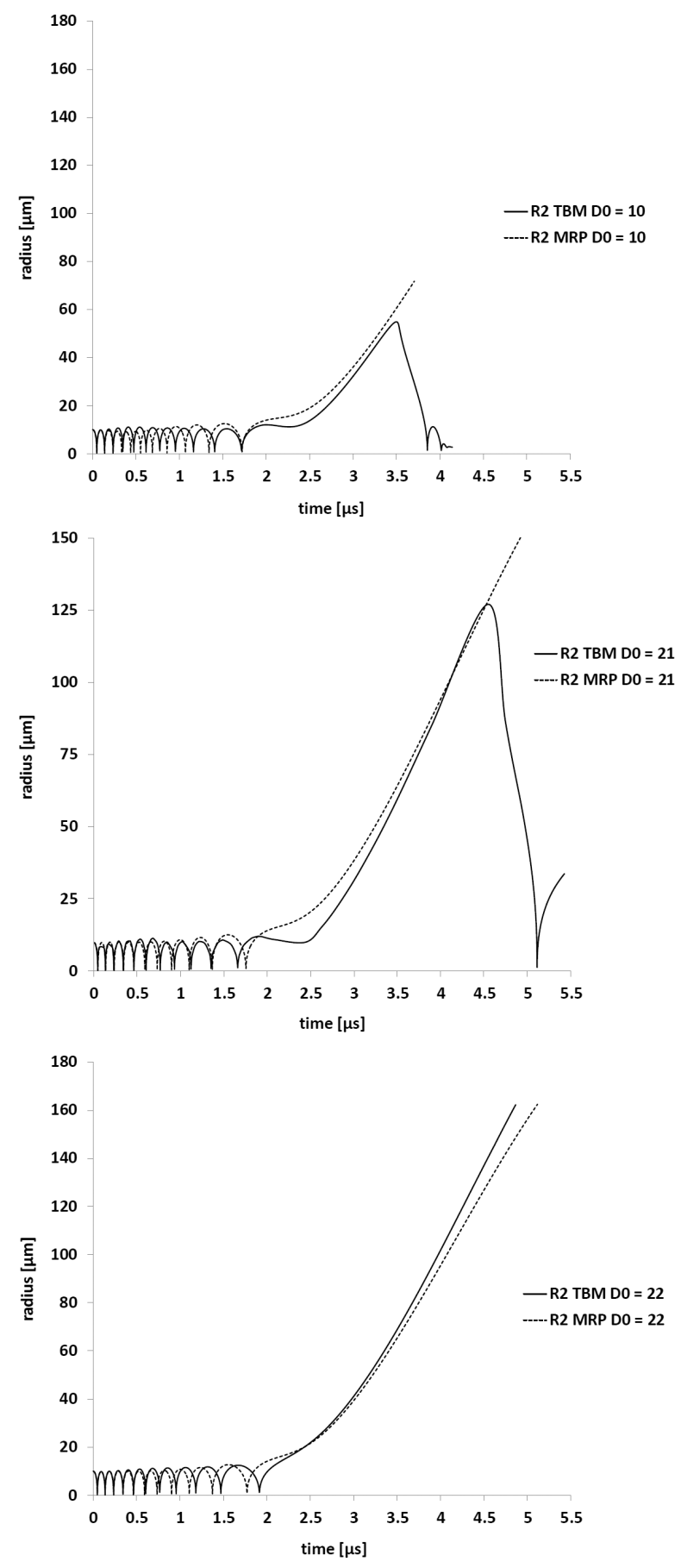

Fig. 11. Comparison of $R_{2}$ from the modified Rayleigh-Plesset model and the two-bubble model for $D_{0}$ equal to 10,21 and 22 . 


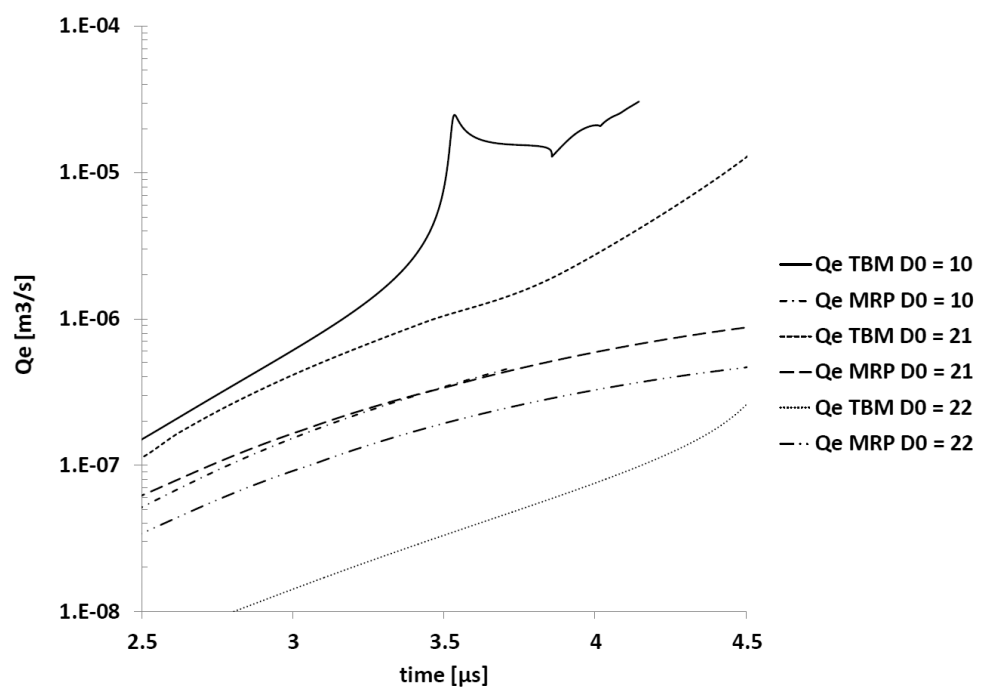

Fig. 12. Evolution of the exchange flow rate $q_{e}$ from the models for $D_{0}$ equal to 10,21 and 22 .

To highlight the importance of the interaction between bubbles by exchange of volume and validate the two-bubble model, the numerical results are compared with the experimental measurements of Ohl [2002]. In the experimental study, bubbles become visible at around $4 \mu \mathrm{s}$. Consequently, only the collapse of small bubbles around this instant interests us. The experimental study lasts about $10 \mu \mathrm{s}$, with certain value of $D_{0}$, the condition $\left(R_{i}+R_{j}\right) \leq 0.75 D_{i j}$ can be reached before $10 \mu \mathrm{s}$. The minimal value of $D_{0}$ which allowed to reach $10 \mu$ s is equal to 31 .

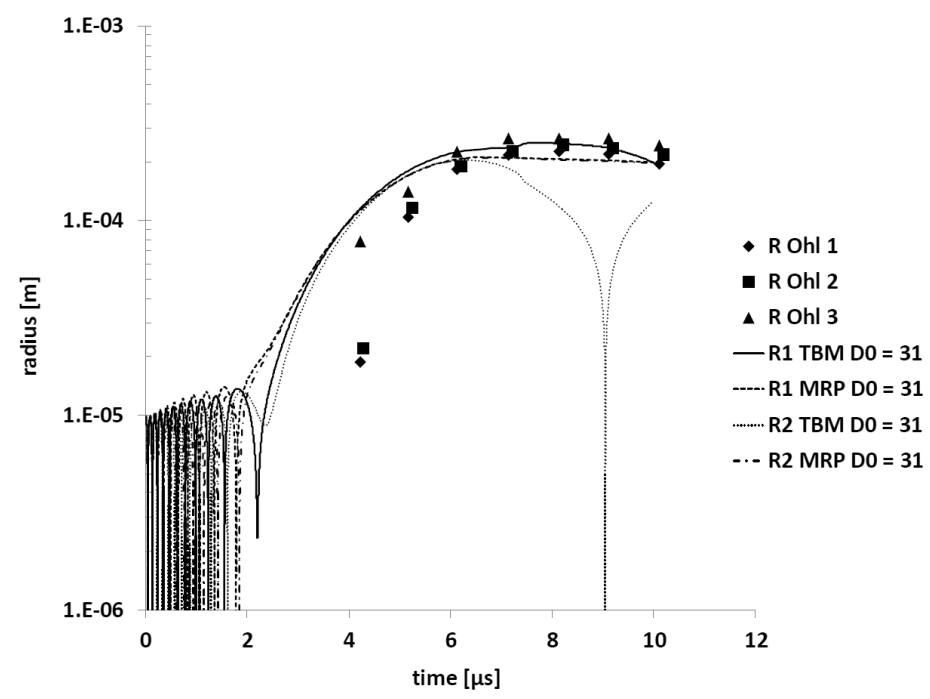

Fig. 13. Evolution of the $R_{1}$ and $R_{2}$ from the models with experimental measurements of Ohl for $D_{0}$ equal to 31 . 


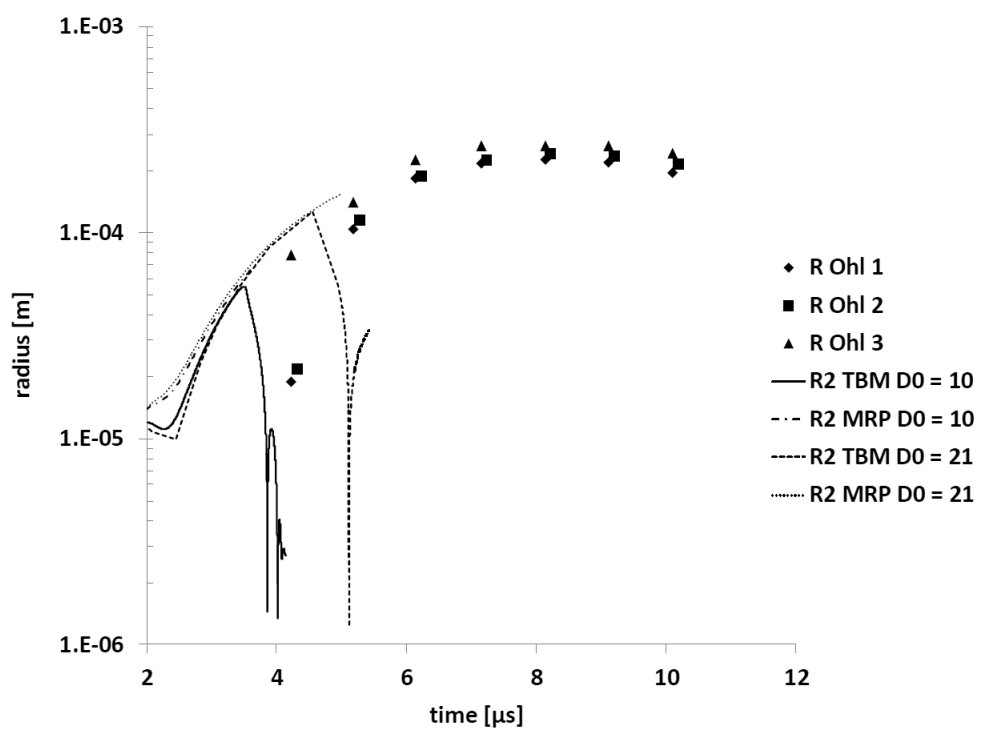

Fig. 14. Evolution of the radius $R_{2}$ from numerical models and experimental measurements of Ohl, for $D_{0}$ equal to 10 and 21 .

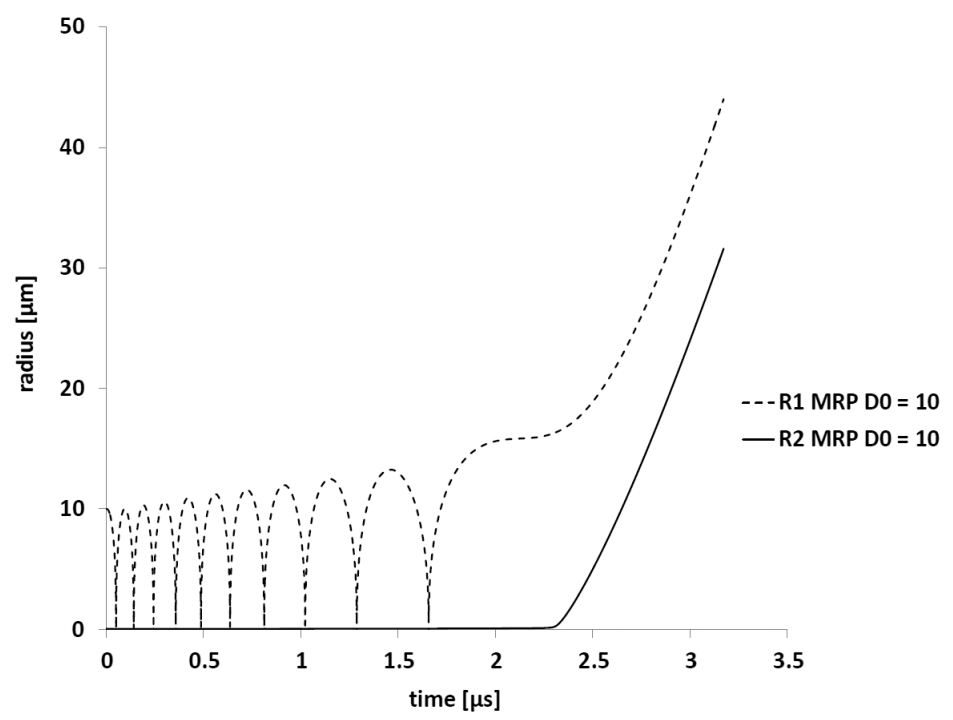

Fig. 15. Evolution of $R_{1}$ and $R_{2}$ from the modified Rayleigh-Plesset model, for $R_{02}=0.1 \mu \mathrm{m}$ and $D_{0}$ equal to 10 .

Figure 13 presents the time evolution of the radii $R_{1}$ and $R_{2}$ of the models compared to experimental measurements of Ohl [2002]. It shows that the small bubble $\left(R_{2}\right)$ continues to grow beyond $4 \mu \mathrm{s}$ in both models, in the modified Rayleigh-Plesset model case it even remains almost equal to the big one. However, in the two-bubble 
model even if the small bubble grows beyond $4 \mu \mathrm{s}$, it finishes by collapsing. This collapse results in an exchange of volume of the small bubble towards the big one.

Concerning the big bubble radius, both models give satisfactory results, with a slight advantage for the two-bubble model, with late collapse of the small bubble. For premature collapses of small bubbles, Fig. 14 shows that, for $D_{0}$ equal to 10 and 21 , the small bubble $\left(R_{2}\right)$ of the two-bubble model develops until it reached the size of the experimental initial bubbles observed before to collapse.

The results indicate particularly that almost all the small bubbles which are in the neighborhood of the big one, for $D_{0}$ less than or equal to 21 , can develop until they reach the size of the experimental initial bubbles observed before the collapse.

With the modified Rayleigh-Plesset model, Fig. 15 demonstrates that even a small bubble of initial radius $R_{02}=0.1 \mu \mathrm{m}$ does not collapse.

\section{Conclusion}

In this study, we were interested in the interaction between bubbles by exchange of volume in the cavitation inception. For a better investigation of the phenomenon, a model with two bubbles is proposed. This two-bubble model is compared to the multibubble model of modified Rayleigh-Plesset and a validation with the experimental measurements of [Ohl, 2002] has been presented. One can conclude that:

The development of the small nearby bubbles can slow down the evolution of the biggest local one and their disappearance can favor the growing of the latter.

During the evolution of two bubbles, the small bubble exchanges volume with the big one. This volume gain allows the big bubble to partially compensate its contraction due to pressure.

With two perfectly identical bubbles, the two-bubble model predicts an exchange of volume between the bubbles equal to zero.

In the validation case with the experimental measurements of Ohl [2002], contrary to the modified Rayleigh-Plesset model, the two-bubble model predicts the appearance and the disappearance of small bubbles in the neighborhood of the big one as it is observed in the experimental study.

The findings show that the interaction by exchange of volume can have an important role in the cavitation born phase. It is also very important to take into account the interaction between bubbles and the disappearance of small bubbles on the evolution of the biggest local one.

\section{Appendix}

In configuration I, 5 bubbles are distributed on a square of side $C_{0}=\sqrt{2} D_{12}$, one of radius $R_{1}$ is situated in the center and four of the same radius $R_{2}$ are placed on each summit: the center bubble of radius $R_{1}$ has 4 bubbles of radius $R_{2}$ at a distance of $D_{12}=\frac{C_{0}}{\sqrt{2}}$. Each summit bubble of radius $R_{2}$ has one bubble of radius $R_{1}$ at a distance of $D_{12}=\frac{C_{0}}{\sqrt{2}}, 2$ of radius $R_{2}$ at a distance of $C_{0}$ and one of radius 
$R_{2}$ at a distance of $\sqrt{2} C_{0}$. So, one can write the following equations:

$$
\begin{aligned}
\dot{q}_{1}= & \frac{1}{8 \pi} \frac{q_{1}^{2}}{R_{1}^{3}}-\frac{4 \mu_{L}}{\rho_{L}} \frac{q_{1}}{R_{1}^{2}}+\frac{4 \pi R_{1}}{\rho_{L}}\left(p_{g_{1}}+p_{v}-p_{\mathrm{ex}}(t)\right)-\frac{8 \pi \sigma}{\rho_{L}}-\frac{4 \sqrt{2} R_{1}}{C_{0}} \dot{q}_{2} \\
\dot{q}_{2}= & \frac{1}{8 \pi} \frac{q_{2}^{2}}{R_{2}^{3}}-\frac{4 \mu_{L}}{\rho_{L}} \frac{q_{2}}{R_{2}^{2}}+\frac{4 \pi R_{2}}{\rho_{L}}\left(p_{g_{2}}+p_{v}-p_{\mathrm{ex}}(t)\right)-\frac{8 \pi \sigma}{\rho_{L}}-\frac{\sqrt{2} R_{2}}{C_{0}} \dot{q}_{1} \\
& -\left(2+\frac{1}{\sqrt{2}}\right) \frac{R_{2}}{C_{0}} \dot{q}_{2} .
\end{aligned}
$$

In configuration II, 9 bubbles are distributed on a cube of edge $C_{0}=\frac{2}{\sqrt{3}} D_{12}$, one of radius $R_{1}$ is located in the center of the cube and 8 of the same radius $R_{2}$ are placed on each summit: the center bubble of radius $R_{1}$ has 8 bubbles of radius $R_{2}$ at a distance of $D_{12}=\frac{\sqrt{3}}{2} C_{0}$. Each summit bubble of radius $R_{2}$ has one of radius $R_{1}$ at a distance of $D_{12}=\frac{\sqrt{3}}{2} C_{0}, 3$ of radius $R_{2}$ at a distance of $C_{0}, 3$ of radius $R_{2}$ at a distance of $\sqrt{2} C_{0}$ and one of radius $R_{2}$ at a distance of $\sqrt{3} C_{0}$. So, one can write the following equations:

$$
\begin{aligned}
\dot{q}_{1}= & \frac{1}{8 \pi} \frac{q_{1}^{2}}{R_{1}^{3}}-\frac{4 \mu_{L}}{\rho_{L}} \frac{q_{1}}{R_{1}^{2}}+\frac{4 \pi R_{1}}{\rho_{L}}\left(p_{g_{1}}+p_{v}-p_{\mathrm{ex}}(t)\right)-\frac{8 \pi \sigma}{\rho_{L}}-\frac{16 R_{1}}{\sqrt{3} C_{0}} \dot{q}_{2} \\
\dot{q}_{2}= & \frac{1}{8 \pi} \frac{q_{2}^{2}}{R_{2}^{3}}-\frac{4 \mu_{L}}{\rho_{L}} \frac{q_{2}}{R_{2}^{2}}+\frac{4 \pi R_{2}}{\rho_{L}}\left(p_{g_{2}}+p_{v}-p_{\mathrm{ex}}(t)\right)-\frac{8 \pi \sigma}{\rho_{L}}-\frac{2 R_{2}}{\sqrt{3} C_{0}} \dot{q}_{1} \\
& -\left(3+\frac{3}{\sqrt{2}}+\frac{1}{\sqrt{3}}\right) \frac{R_{2}}{C_{0}} \dot{q}_{2} .
\end{aligned}
$$

In configuration III, we consider 15 bubbles distributed in a cube of edge $C_{0}=$ $2 D_{12}$, one of radius $R_{1}$ is situated in the center of the cube, 6 bubbles of the same radius $R_{2}$ are placed in the center of each face and 8 bubbles of the same radius $R_{3}$ are located on each summit: the center bubble of radius $R_{1}$ has 6 bubbles of radius $R_{2}$ at a distance of $D_{12}=\frac{1}{2} C_{0}$ and 8 of radius $R_{3}$ at a distance of $D_{13}=\frac{\sqrt{3}}{2} C_{0}$. Each face bubble of radius $R_{2}$ has one bubble of radius $R_{1}$ at a distance of $D_{12}=\frac{1}{2} C_{0}$, 4 of radius $R_{3}$ at a distance of $\frac{\sqrt{2}}{2} C_{0}, 4$ of radius $R_{3}$ at a distance of $\sqrt{3 / 2} C_{0}, 4$ of radius $R_{2}$ at a distance of $\frac{C_{0}}{\sqrt{2}}$ and one of radius $R_{2}$ at a distance of $C_{0}$. Each summit bubble of radius $R_{3}$ has one of radius $R_{1}$ at a distance of $D_{13}=\frac{\sqrt{3}}{2} C_{0}, 3$ of radius $R_{2}$ at a distance of $\frac{\sqrt{2}}{2} C_{0}, 3$ of radius $R_{2}$ at a distance of $\sqrt{3 / 2} C_{0}, 3$ of radius $R_{3}$ at a distance of $C_{0}, 3$ of radius $R_{3}$ at a distance of $\sqrt{2} C_{0}$ and one of radius $R_{3}$ at a distance of $\sqrt{3} C_{0}$. So, one can write the following equations:

$$
\begin{aligned}
\dot{q}_{1}= & \frac{1}{8 \pi} \frac{q_{1}^{2}}{R_{1}^{3}}-\frac{4 \mu_{L}}{\rho_{L}} \frac{q_{1}}{R_{1}^{2}}+\frac{4 \pi R_{1}}{\rho_{L}}\left(p_{g_{1}}+p_{v}-p_{\mathrm{ex}}(t)\right) \\
& -\frac{8 \pi \sigma}{\rho_{L}}-\frac{12 R_{1}}{C_{0}} \dot{q}_{2}-\frac{16 R_{1}}{\sqrt{3} C_{0}} \dot{q}_{3}
\end{aligned}
$$




$$
\begin{aligned}
\dot{q}_{2}= & \frac{1}{8 \pi} \frac{q_{2}^{2}}{R_{2}^{3}}-\frac{4 \mu_{L}}{\rho_{L}} \frac{q_{2}}{R_{2}^{2}}+\frac{4 \pi R_{2}}{\rho_{L}}\left(p_{g_{2}}+p_{v}-p_{\mathrm{ex}}(t)\right)-\frac{8 \pi \sigma}{\rho_{L}}-\frac{2 R_{2}}{C_{0}} \dot{q}_{1} \\
& -(4 \sqrt{2}+1) \frac{R_{2}}{C_{0}} \dot{q}_{2}-\left(\frac{8}{\sqrt{2}}+4 \frac{\sqrt{2}}{\sqrt{3}}\right) \frac{R_{2}}{C_{0}} \dot{q}_{3} \\
\dot{q}_{3}= & \frac{1}{8 \pi} \frac{q_{3}^{2}}{R_{3}^{3}}-\frac{4 \mu_{L}}{\rho_{L}} \frac{q_{3}}{R_{3}^{2}}+\frac{4 \pi R_{3}}{\rho_{L}}\left(p_{g_{3}}+p_{v}-p_{\mathrm{ex}}(t)\right)-\frac{8 \pi \sigma}{\rho_{L}}-\frac{2 R_{3}}{\sqrt{3} C_{0}} \dot{q}_{1} \\
& -\left(\frac{6}{\sqrt{2}}+3 \frac{\sqrt{2}}{\sqrt{3}}\right) \frac{R_{3}}{C_{0}} \dot{q}_{2}-\left(3+\frac{3}{\sqrt{2}}+\frac{1}{\sqrt{3}}\right) \frac{R_{3}}{C_{0}} \dot{q}_{3}
\end{aligned}
$$

\section{References}

Abe, H., Ueda, T., Saitoh, Y., Nomura, R., Okuda, Y. and Burmistrov, S. N. [2007] "Visual observation of a sound-induced bubble in liquid ${ }^{3} \mathrm{He}-{ }^{4} \mathrm{He}$ mixtures," Journal of Low Temperature Physics 148, 133-138.

Arndt, R., Voigt, R. Jr., Sinclair, J., Rodrique, P. and Ferreira, A. [1989] "Cavitation Erosion in Hydroturbines," Journal of Hydraulic Engineering 115(10), 1297-1315.

Astolfi, J.-A., Dorange, P., Billard, J.-Y. and Cid Tomas, I. [2000] "An experimental investigation of cavitation inception and development on a two-dimensional eppler hydrofoil," Journal of Fluids Engineering 122, 164-173.

Bremond, N., Arora, M., Ohl, C. D. and Lohse, D. [2005] "Cavitation on surfaces," Journal of Physics: Condensed Matter 17, S3603-S3608.

Bremond, N., Arora, M., Dammer, S. M. and Lohse, D. [2006] "Interaction of cavitation bubbles on a well," Physics of Fluids 18, 1215505.

Bremond, N., Arora, M., Ohl, C. D. and Lohse, D. [2006] "Controlled multibubble surface cavitation," Physical Review Letters 96, 224501.

Buogo, S. and Cannelli, G. B. [2002] "Implosion of an underwater spark-generated bubble and acoustic energy evaluation using the Rayleigh model," The Journal of the Acoustical Society of America 111(6), 2594-2599.

Chahine, G. L. [1984] "Pressures generated by a bubble cloud collapse," Chemical Engineering Communications 28, 355-367.

Coutier-Delgosha, O., Stutz, B., Vabre, A. and Legoupil, S. [2007] "Analysis of cavitating flow structure by experimental and numerical investigations," Journal of Fluid Mechanics 578, 171-222.

D'Agostino, L. and Brennen, C. E. [1989] "Linearized dynamics of spherical bubbly clouds," Journal of Fluid Mechanics 199, 155-176.

Doinikov, A. A. [2001] "Translational motion of two interacting bubbles in a strong acoustic field," Physical Review E 64, 026301.

Fortes-Patella, R., Reboud, J.-L. and Archer, A. [2000] "Cavitation damage measurement by 3D laser profilometry," Wear 246, 59-67.

Franc, J.-P. and Michel, J.-M. [1985] "Attached cavitation and the boundary layer: Experimental investigation and numerical treatment," Journal of Fluid Mechanics 154, 63-90.

Gilmore, F. R. [1952] "The growth or collapse of a spherical bubble in a viscous compressible liquid," California Institute of Technology, Hydrodynamics Laboratory, Report 26-4. 
Hajizadeh Aghdam, A., Ohl, S. W., Khoo, B. C., Shervani-Tabar, M. T. and Nobari, M. R. H. [2012] "Effect of the viscosity on the behavior of a single bubble near a membrane," International Journal of Multiphase Flow 47(9), 17-24.

Harkin, A., Kaper, T. J. and Nadim, A. [2001] "Coupled pulsation and translation of two gas bubbles in a liquid," Journal of Fluid Mechanics 445, 377-411.

Ida, M., Naoe, T. and Futakawa, M. [2007] "Suppression of cavitation inception by gas bubble injection: A numerical study focusing on bubble-bubble interaction," Physical Review E 76, 046309.

Ida, M. [2009] "Multibubble cavitation inception," Physics of Fluids 21, 113302.

Keller, K. B. and Miksis, M. [1980] "Bubble oscillations of large amplitude," The Journal of the Acoustical Society of America 68, 628.

Kubota, A., Kato, H. and Yamaguchi, H. [1992] "A new modelling of cavitating flows: A numerical study of unsteady cavitation on a hydrofoil section," Journal of Fluid Mechanics 240, 59-96.

Lauterborn, W. and Bolle, H. [1975] "Experimental investigations of cavitation-bubble collapse in neighborhood of a solid boundary," Journal of Fluid Mechanics 72, 391.

Madadnia, J. and Owen, I. [1995] "Erosion in conical diffusers in particulate-laden cavitating flow," International Journal of Multiphase Flow 21(6), 1253-1257.

Mettin, R., Akhatov, I., Parlitz, U., Ohl, C. D. and Lauterborn, W. [1997] "Bjerknes forces between small cavitation bubbles in a strong acoustic field," Physical Review E 56(3), $2924-2931$.

Oguz, H. N. and Prosperetti, A. [1990] "A generalization of the impulse and virial theorems with an application to bubble oscillations," Journal of Fluid Mechanics 218, 143-162.

Ohl, C. D. [2002] "Cavitation inception following shock wave passage," Physics of Fluids 14(10), 3512-3521.

Plesset, M. S. [1948] "Dynamics of cavitation bubbles," Journal of Applied Mechanics 16, $228-231$.

Rayleigh, L. [1917] "On the pressure developed in a liquid during the collapse of a spherical cavity," Philosophical Magazine 34(200), 94-98.

Stutz, B. and Legoupil, S. [2003] "X-ray measurements within unsteady cavitation," Experiments in Fluids 35, 130-138.

Takahira, H., Akamatsu, T. and Fujikawa, S. [1994] "Dynamics of a Cluster of Bubbles in a Liquid," JSME International Journal Series B 37, 297-305.

Yang, S.-H., Jaw, S.-Y. and Yeh, K.-C. [2009] "Single cavitation bubble generation and observation of the bubble collapse flow induced by a pressure wave," Experiments in Fluids 47, 343-355. 